\title{
Kinetics of Norepinephrine- and Serotonin-Induced BDNF Release in Cultured Embryonic Hippocampal Neurons"
}

\author{
Michael Chen, Amelia Russo-Neustadt \\ Department of Biological Sciences, California State University, Los Angeles, USA. \\ Email: mchen@calstatela.edu
}

Received August 22 $2^{\text {nd }}, 2013$; revised September $20^{\text {th }}, 2013$; accepted October $15^{\text {th }}, 2013$

Copyright (C) 2013 Michael Chen, Amelia Russo-Neustadt. This is an open access article distributed under the Creative Commons Attribution License, which permits unrestricted use, distribution, and reproduction in any medium, provided the original work is properly cited.

\begin{abstract}
The primary neurotransmitters targeted by currently used antidepressants, such as duloxetine, venlafaxine and fluoxetine, are serotonin and norepinephrine, which also are released in significant amounts in the central nervous system in response to sympathetic nervous system activation. In cultured hippocampal neurons, we have previously shown that norepinephrine induces increased expression of brain-derived neurotrophic factor (BDNF), the PI-3 K/Akt, MAPK pro-survival pathways, the BDNF receptor, TrkB, a transcription factor, and cyclic AMP response element binding protein (CREB). In the present study, we extend these findings of increased BDNF expression to its kinetics of release into the surrounding media. We also evaluate these two cell survival pathways, TrkB and CREB, in response to application of serotonin and/or norepinephrine. Serotonin elicits an earlier, but brief expression and release of BDNF, whereas norepinephrine elicits a more delayed and sustained release of BDNF. In response to both norepinephrine and 5-HT, the presence of BDNF in lysates and subsequent release into the media is significantly increased out to $4 \mathrm{~h}$, as is PI-3 K/Akt activation. Together, these two neurotransmitters increase BDNF expression and release covering the entire $8 \mathrm{~h}$ continuum evaluated. The results of this study provide further evidence for a $\mathrm{G}$ protein-coupled receptor and a crosstalk-toTrkB receptor with transactivation signaling across pathways.
\end{abstract}

Keywords: BDNF; CREB; Serotonin; Norepinephrine; Hippocampus; Kinetics

\section{Introduction}

Neuronal activity that increases as a result of sympathetic nervous system stimulation [1], such as physical activity [2-4] or administration of antidepressant drugs [5], has been shown to increase levels of peripheral [6] or CNS [4, 7-9] brain-derived neurotrophic factor (BDNF), and whether that activity is electrical stimulation $[1,10]$, extracellular application of $\mathrm{K}^{+}[1,11-17]$ or $\mathrm{Ca}^{2+}$ [13] or application of excitatory neurotransmitter (e.g., kainic acid, [11,15, 18-21]), forskolin (cAMP activator [15]) or inhibitory neurotransmitter antagonist (CGP 52432, $\mathrm{GABA}_{\mathrm{B}}$ recaptor antagonist [15]). Conversely, anything that blocks neurotransmission, such as tetrodotoxin $\left(\mathrm{Na}^{+}\right.$channel blocker $[10,12,22]$ ), nifedipine $[11,22]$, BAPTA-AM (intracellular $\mathrm{Ca}^{2+}$ chelator [12]) pertussis toxin (G-protein inhibitor [23]), Y 25130 (5- $\mathrm{HT}_{3}$ antagonist [15]) or AMPA or NMDA receptor blocker [21], has been shown to de-

*Funding was provided by PHS grant MH 559776 to ARN.

The authors declare no conflict of interest. crease BDNF secretion.

We have recently shown that BDNF levels in cultured hippocampal neurons will increase in response to application of norepinephrine [24-26]. Such cultured conditions were initially designed to model any activity or intervention in vivo [27] that results in greater release of norepinephrine, which has been shown to be neuroprotective by increasing expression, secretion and release of BDNF $[7,28]$. The putative purpose of antidepressants is to inhibit the reuptake or metabolism of 5-HT or norepinephrine; although it is not yet definitively known why the therapeutic efficacy is such that these drugs take 2 - 4 weeks to work, the prevailing thinking suggests that changes in gene expression require such a time scale $[29,30]$.

In our hippocampal tissue culture model, application of norepinephrine elicits maximum BDNF expression at 2 hours [24]. In the present study, we extend these previous results obtained with norepinephrine and subse- 
quent activation of the survival/neuroprotective PI-3 K/ Akt and MAPK pathways [24] to determine how long 5HT takes in eliciting maximum BDNF expression and secretion and signaling of these same two pathways.

\section{Materials and Methods}

\subsection{Drugs and Chemicals}

Norepinephrine, serotonin (5-HT), PD 98059, LY 294002 and wortmannin were purchased from Sigma Chemical Co. (St. Louis, MO). K252a was purchased from Calbiochem (La Jolla, CA). Dulbecco's modified eagle medium (DMEM) was purchased from ATCC (Manassas, VA). N 2 was purchased from Invitrogen (Eugene, OR). The BDNF $\mathrm{E}_{\max }$ Immunoassay System was purchased from Promega (Madison, WI). PD 98059 and wortmannin were initially dissolved in DMSO; at no time did the concentration of DMSO exceed $0.5 \%$ of the total volume in each tissue culture well.

\subsection{Antibodies}

Anti-BDNF was purchased from Santa Cruz Biotech (Santa Cruz, CA). Anti-phospho-CREB, anti-CREB, anti-phospho-T 308-Akt, anti-Akt, anti-phospho TrkA (Y 490), which cross-reacts with anti-phospho-TrkB, and anti-TrkB were purchased from Cell Signaling Technology (Danvers, MA). Anti-phospho-MAPK, anti-MAPK, and anti-tubulin were purchased from Millipore (Temecula, CA). Secondary antibodies, anti-rabbit IgG and antimouse IgG, were purchased from Amersham-Pharmacia Biotech (Piscataway, NJ).

\subsection{Animals: Ethics Statement}

All efforts were made to minimize the number of animals used. We have abided by the use of the ethical treatment of laboratory animals as specified in the National Research Council's Guide for the Care and Use of Laboratory Animals (1996). The Institutional Animal Care and Use Committee (IACUC) and the Institutional Review Board-Human Subjects at California State University, Los Angeles, approved this project. The IACUC Protocol number is AW $10-1$.

\subsection{Hippocampal Dissection at Embryonic Day 18 (E 18)}

Female rats (Sprague-Dawley, Charles River Corp., Wilmington, MA) were sacrificed with an overdose of isofluorane on their $18^{\text {th }}$ day of pregnancy. The embryos were removed by cesarean section and immediately placed on ice. The two hippocampi of each embryo were excised and cultured in $\mathrm{Ca}-\mathrm{Mg}$-free medium according to the method of Banker and Cowan [31]. The dissected hippocampi were then rinsed twice with $5-10 \mathrm{ml} /$ rinse of Ca-Mg-free medium in 15-ml conical tubes. Trypsin $(0.125 \%)$ was added to the Ca-Mg-free medium ( $4-5$ $\mathrm{ml})$ and then the conical tube was placed in a water bath at $37^{\circ} \mathrm{C}$ for $5-10 \mathrm{~min}$ with gentle inversion every $2-3$ min. Cells were quenched in $10 \%$ fetal bovine serum/ DMEM (warmed to $37^{\circ} \mathrm{C}, 1-2$ volume $\sim 8 \mathrm{ml}$ ) to stop the reaction and centrifuged at $200 \mathrm{~g}$ for $5 \mathrm{~min}$. The supernatant was then removed and the pellet resuspended in $\sim 2 \mathrm{ml}$ serum-free DMEM. Neurons were then triturated (pipetting up and down mechanically for separation) with a silicote-coated constricted glass pipette and filtered through a $40 \mu \mathrm{m}$ cell strainer (Falcon) into a sterile $50 \mathrm{ml}$ conical tube. The volume was then supplemented with serum-free $1 \% \mathrm{~N} 2 / \mathrm{DMEM}$ to a total volume of 5 ml. Trypan blue $(10 \mu \mathrm{l})$ was then added to $10 \mu \mathrm{l}$ of cells, which were counted using a hemacytometer. Neurons were then plated in poly-1-lysine-coated plates at a density of 50,000 cells $/ \mathrm{cm}^{2}$. Treatment of hippocampal mixed neuronal/astrocytic cultures was conducted in triplicate where each experiment was performed at least twice. Cells were then placed in an incubator at $37^{\circ} \mathrm{C}$ and $5 \% \mathrm{CO}_{2}$.

\subsection{5-HT Dose-/Time Response Evaluation}

On the $3^{\text {rd }}-4^{\text {th }}$ day following plating, 5-HT was added to the appropriate wells in triplicate at concentrations of $10^{-9}, 10^{-8}, 10^{-7}, 10^{-6}, 10^{-5}$, and $10^{-4} \mathrm{M}$ and allowed to incubate at $37^{\circ} \mathrm{C}, 5 \% \mathrm{CO}_{2}$, for $0,5,10,30,60$, and 120 min, culminating in 72 combination treatments. These concentrations and time points were partially guided by experiments performed by Cowan et al. [32]. At each of these time points, the media was aspirated off and the cells in each respective well lysed with the addition of 75 $\mu \mathrm{l}$ lysis buffer (10 mM tris, $\mathrm{pH} 7.4,1 \%$ SDS), scraped from their wells, boiled for $5 \mathrm{~min}$, triturated through a 26-gauge needle, centrifuged at $14,000 \mathrm{~g}$ for $5 \mathrm{~min}$, and an equal volume of gel-loading buffer [33] added to the supernatant. Samples were stored at $-80^{\circ} \mathrm{C}$ until ready for SDS-PAGE/Western Blotting.

\subsection{Norepinephrine, 5-HT or the Combination}

On the third day following plating, norepinephrine $\left(10^{-7}\right.$ $\mathrm{M}$ [24]) and/or 5-HT $\left(10^{-5} \mathrm{M}\right)$ was added to the appropriate treatment wells, and plates allowed to incubate at $37^{\circ} \mathrm{C}, 5 \% \mathrm{CO}_{2}$, for $10,30,60,120,240$, or $480 \mathrm{~min}$ (with norepinephrine only or norepinephrine +5 -HT) or for 0 , $5,10,30,60,120$, or $240 \mathrm{~min}$ (with 5 -HT only). At each of these time points, three $100 \mu$ laliquots (triplicate) of media was withdrawn from each well and stored at $-20^{\circ} \mathrm{C}$ till assaying with BDNF ELISA kit. At the same respective time point, the rest of the media was aspirated off and the cells in each respective well processed for SDS-PAGE just as described in the preceding section. 


\subsection{SDS-PAGE/Western Blotting}

Equal amounts of cell lysates were applied to each lane of an SDS-PAG, electrophoresed, and the separated proteins subsequently electro-transferred to nitrocellulose (Amersham-Pharmacia Biotech, Piscataway, NJ). Western blotting on nitrocellulose membranes was performed according to the specifications of the respective manufacturers. Protein immunoreactivity was visualized by enhanced chemiluminescence (ECL), followed by apposition of blots to hyperfilm (Amersham-Pharmacia Biotech, Piscataway, NJ). To control for inadvertent differences in protein loading, all blots were then stripped in $100 \mathrm{mM}$ 2-mercaptoethanol, 2\% SDS, $62.5 \mathrm{mM}$ tris-HCl, $\mathrm{pH} 6.7$, at $55^{\circ} \mathrm{C}$ for $10 \mathrm{~min}$, with agitation and then reprobed with the antibody against the respective total form (anti-Akt, anti-MAPK, or anti-CREB), followed by ECL and apposition to hyperfilm. Blots first probed with anti-BDNF were stripped and then re-probed with antitubulin. Blots first probed with the phospho-forms, followed by the total forms, were then stripped once more and re-probed with anti-tubulin. All anti-tubulin reactions were then reacted once again with ECL and apposed to hyperfilm.

Optical densities of lightly exposed phospho-proteins, BDNF, total forms (e.g., total CREB), and tubulin bands corresponding to the relative mobility of each respective signaling protein were quantified using computer-assisted densitometry (MCID, Interfocus Imaging Ltd, London). Optical densities of the phospho-forms were then arithmetically divided by those of their respective total forms; this quotient was then arithmetically divided by the respective tubulin band. Optical density of BDNF bands was arithmetically divided by those of their respective tubulin bands. All exposed bands quantified within the linear range of a standard curve were used in gathering densitometry data.

\subsection{BDNF ELISA}

The presence of BDNF in the media in response to norepinephrine, 5-HT, or both at each time point was detected using the BDNF $\mathrm{E}_{\max }$ Immunoassay System (Promega, Madison, WI).

\subsection{Statistical Analyses}

For the Western data generated from the initial 5-HT dose-and time-response evaluation of BDNF immunoreactivity, a 2-way ANOVA (dose $\times$ time) was calculated, using Fisher's post hoc LSD to evaluate pair-wise comparisons. For both the Western blot-generated optical densities and the BDNF $\mathrm{E}_{\max }$ data, statistical analyses were implemented using 1-way ANOVA, followed by Fisher's post hoc LSD to test for pair-wise statistically significant differences between any two time points. For analyzing how the activity of intracellular signaling molecules changes with respect to each other and over time, a 2-way ANOVA (molecule $\times$ time), followed by Fisher's post hoc LSD test was conducted. For analyzing how the levels of BDNF change with respect to each other and over time, a 2-way ANOVA (condition $\times$ time), followed by Fisher's post hoc LSD test was conducted, where condition is either lysates or media.

\section{Results}

\subsection{Dose- and Time-Response of BDNF Immunoreactivity to 5-HT in Hippocampal Cell Lysates}

Application of 5-HT to E 18 hippocampal neurons significantly induced BDNF immunorectivity in a dose- $\left[\mathrm{F}_{(6}\right.$, $\left.{ }_{84)}=138.38, p<0.0001\right]$ and time $-\left[\mathrm{F}_{(5,84)}=736.65, p<\right.$ $0.0001]$ dependent manner. In addition, there was significant interaction between these two variables $\left[\mathrm{F}_{(30,84)}\right.$ $=35.45, p<0.0001]$ (Figure 1). BDNF immunoreactivity was increased significantly in response to only $10^{-9}$ M 5-HT in as little time as $5 \mathrm{~min}(p<0.0001)$ and continuing through to $10 \mathrm{~min}(p<0.0001)$, compared to that of vehicle-treated controls at the corresponding zero time-points of 5 and $10 \mathrm{~min}$ (Figure 1). This same trend was also evident for all 5 of the higher doses evaluated $\left(10^{-8}, 10^{-7}, 10^{-6}, 10^{-5}\right.$, and $10^{-4} \mathrm{M} 5$-HT $)$, compared to vehicle-treated controls and compared to the zero time point. However, at $10^{-7} \mathrm{M} 5$-HT, BDNF immunoreactivity was significantly increased at $30 \mathrm{~min}$, compared to vehicletreated controls and compared to the zero time point, but then decreased to levels comparable to those of controls (vehicle-treated at zero $\mathrm{min}$ ) at 60 through to

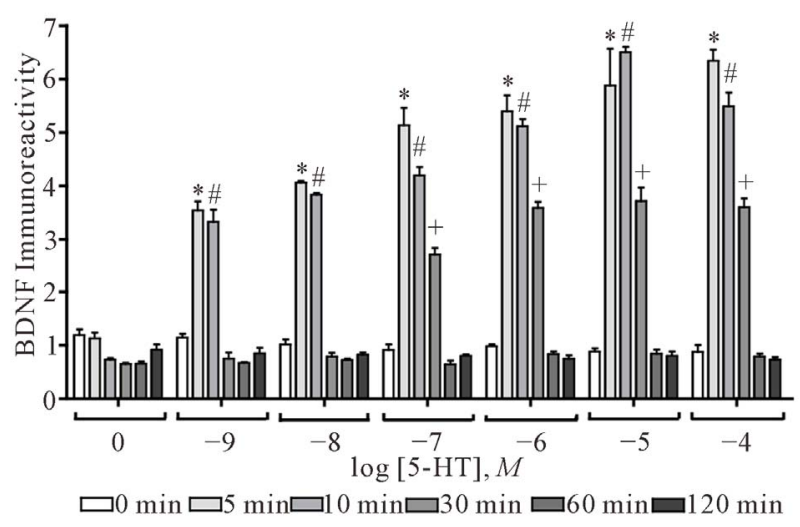

Figure 1. Serotonin elicits BDNF immunoreactivity in hippocampal neurons in a dose- and time-dependent manner. *, statistically significantly different from vehicle controls ([5$\mathrm{HT}]=\mathbf{0} \mathrm{M}$ at $5 \mathrm{~min}(p<0.001)$; \#, statistically significantly different from vehicle controls $([5-\mathrm{HT}]=0 \mathrm{M}$ at $10 \mathrm{~min}(p<$ $0.001) ;+$, statistically significantly different from vehicle controls $([5-\mathrm{HT}]=0 \mathrm{M}$ at $30 \mathrm{~min}(p<0.001)$. Data are the mean \pm SEM. Each experiment was conducted at least twice, with each sample measured in triplicate. 
$120 \min$ (Figure 1).

\subsection{Kinetics of BDNF Release into the Media in Response to 5-HT, Norepinephrine, and Both}

As per the results displayed in Figure 1, the rest of this study was performed with $10^{-5} \mathrm{M} 5$-HT, as this concentration was one of the two that yielded the most BDNF immunoreactivity (the other being $10^{-4} \mathrm{M}$ ). Norepinephrine was applied at a final concentration of $10^{-7} \mathrm{M}$, as this dose has been shown to elicit the highest BDNF immunoreactivity in a similar dose-/time-dependent experiment [24].

Application and incubation of the aforementioned concentrations of 5-HT $+/-$ norepinephrine at 5, 10, 30, 60, 120, 240, and 480 min display the results in Figure 2. There was a statistically significant interaction $\left[\mathrm{F}_{(13,27)}=\right.$ 13.23, $p<0.0001]$ between time point and neurotransmitter (5-HT, norepinephrine, both, or neither (vehicle controls)) and a significant main effect of neurotransmitter $\left[\mathrm{F}_{(3,27)}=76.66, p<0.0001\right]$, but not for time point.

For each time point up to and including $120 \mathrm{~min}$, norepinephrine-induced secretion of BDNF was no different from that of vehicle; but at $240 \mathrm{~min}$, norepinephrine lead to significantly more BDNF secretion into the media than that of vehicle $(p=0.002)$. In sharp contrast, however, 5-
HT led to significantly more BDNF secretion than that of vehicle much earlier at $10(p<0.0001), 30(p=0.004)$ and $60(p=0.015) \mathrm{min}$. At 120 and $240 \mathrm{~min}$, however, vehicle- and 5-HT-secreted BDNF values were comparable (Figure 2). When both neurotransmitters were combined, however, all time points revealed significantly more BDNF secretion into the media than that of vehicle alone $[5 \min (p<0.0001), 10 \mathrm{~min}(p<0.0001), 30 \mathrm{~min}$ $(p=0.051), 60 \min (p=0.002), 120 \min (p<0.0001)$, $240 \min (p<0.0001)$ ] (Figure 2).

Comparison of the amount of BDNF secretion between vehicle and either neurotransmitter or both: veh vs. 5-HT [10 $\min (p<0.000), 30 \min (p=0.004), 60 \min (p$ $=0.015)]$; veh vs. norepinephrine $[240 \mathrm{~min}(p=0.002)$, $480 \min (p=0.006)]$; veh vs. norepinephrine +5 -HT $(p$ $<0.0001-0.05)$. And comparison of the amount of BDNF secretion between either neurotransmitter alone and that of the combination treatment resulted in either statistically significant differences or a trend (Figure 2): 5-HT vs. norepinephrine +5 -HT $[10 \min (p=0.057), 30$ $\min (p=0.031), 60 \min (p=0.056), 120 \min (p<$ $0.0001), 240 \mathrm{~min}(p=0.016)]$; norepinephrine vs. norepinephrine +5 -HT $[10 \min (p<0.0001), 30 \min (p=$ $0.03), 60 \min (p=0.002), 120 \min (p=0.001), 240 \mathrm{~min}$ $(p=0.016), 480 \min (p=0.011)]$ (Figure 2).

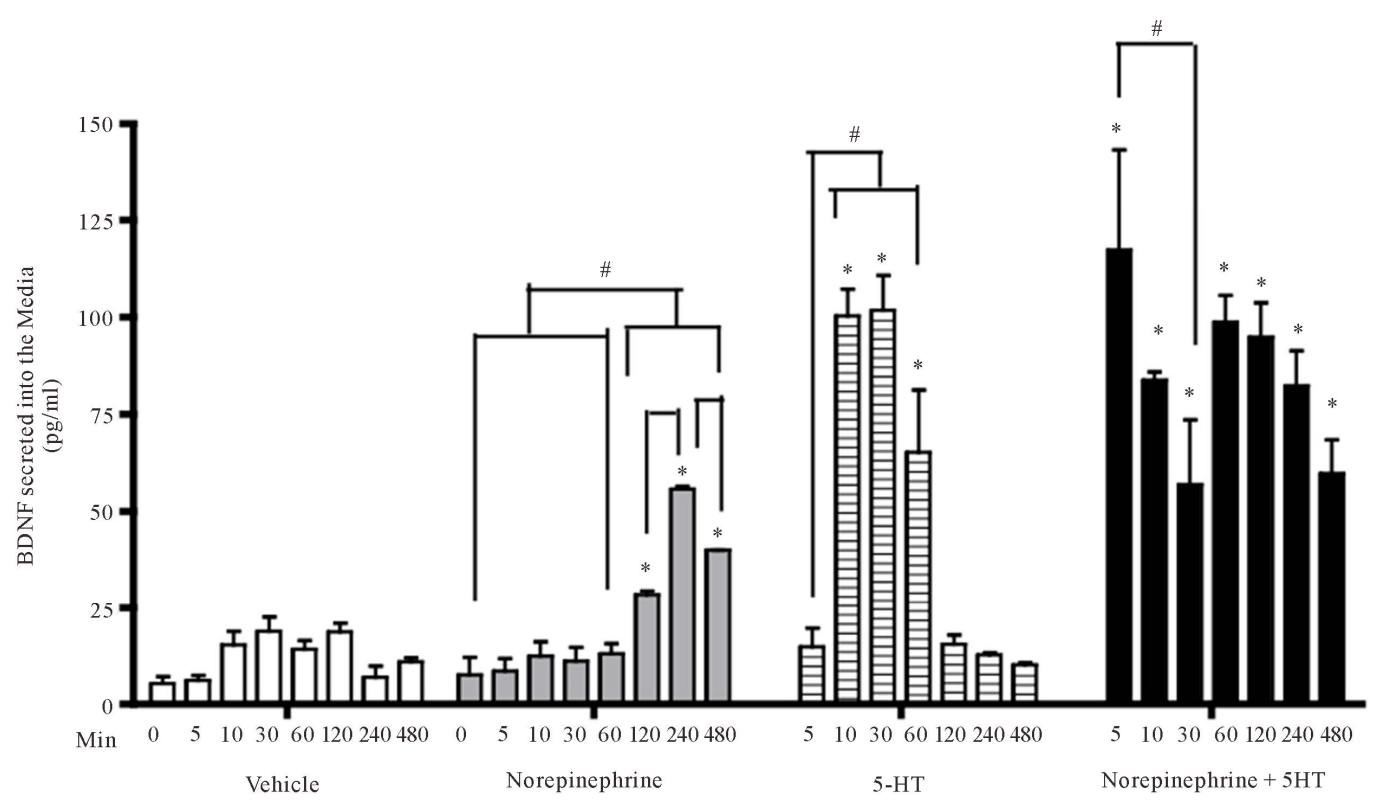

Figure 2. Serotonin and/or norepinephrine elicit the secretion of BDNF into the media from hippocampal neurons in a doseand time-dependent manner. Serotonin caused significantly more BDNF secretion at 10, 30, and 60 min than at 5 min $(p \leq$ $0.005)$; norepinephrine elicited significantly more BDNF secretion at $120 \mathrm{~min}$ than at $5,10,30$, and $60 \mathrm{~min}(p \leq 0.004)$; norepinephrine elicited significantly more BDNF secretion at 240 min than at either $120(p<0.0001)$ or $480(p<0.001)$ min; and both neurotransmitters combined elicited as much as 2 - 5 times the amount elicited by vehicle alone at all time points. Among the combination treatment itself, there was a statistically significant decrease in BDNF secretion at 30 min, compared to that at $5 \mathrm{~min}(p=0.022)$ and a lower, but not statistically significantly lower, than that at $60 \mathrm{~min}(p=0.077)$ *, statistically significantly different from that of vehicle controls at the same corresponding time point at $p<0.05$. \#: significantly different at $p<0.05$. Data are the mean \pm SEM. Each experiment was conducted at least twice, with each sample measured in triplicate. 


\subsection{Kinetics of Activation of Intracellular Signaling in Hippocampal Cell Lysates in Response to 5-HT}

Both the PI-3 K and MAPK pathways were differentially activated in response to application of 5-HT over time (Figure 3). PI-3 K was significantly activated over time $\left[\mathrm{F}_{(6,17)}=7.2, p=0.001\right]$. Only at $30(p=0.007)$ and $60(p$ $<0.000)$ min was PI-3 K expression significantly higher than that at the zero time point (Figure 3(a)); PI-3 K expression at $30 \mathrm{~min}$ was significantly higher than that at $240 \min (p=0.015)$. Phosphorylation of Akt exhibited overall statistical significance over time $\left[\mathrm{F}_{(6,17)}=6.5, p=\right.$ $0.015]$ with its activation being significantly higher at 30 $(p=0.026)$ and $60(p=0.041)$ min than at zero $\min$ (Figure 3(a)).

BDNF expression was significantly activated in response to application of 5-HT over time $\left[\mathrm{F}_{(5,12)}=70.83\right.$, $p<0.000]$, where the 5-, 10- and 30-min time points were all significantly higher than that at the zero-min time point $(p<0.000)$; BDNF expression at 5 min was significantly higher than at all other time points, except that at $10 \min (p<0.000)$; the 10 -min time point was significantly higher than at the 30-, 60-, and 120-min time points $(p<0.000)$; finally, BDNF expression was significantly higher at the 5-, 10-, and 30-min time points than that at $60 \min (p<0.000)$ (Figure 3(b)).

Phosphorylation of the BDNF receptor, TrkB, was not overall significant over time, although the 240-min time point exhibited statistically significantly higher phosphorylation levels than that at zero $(p=0.012)$ and 60 $\min (p=0.03)$ (Figure 3(b)).

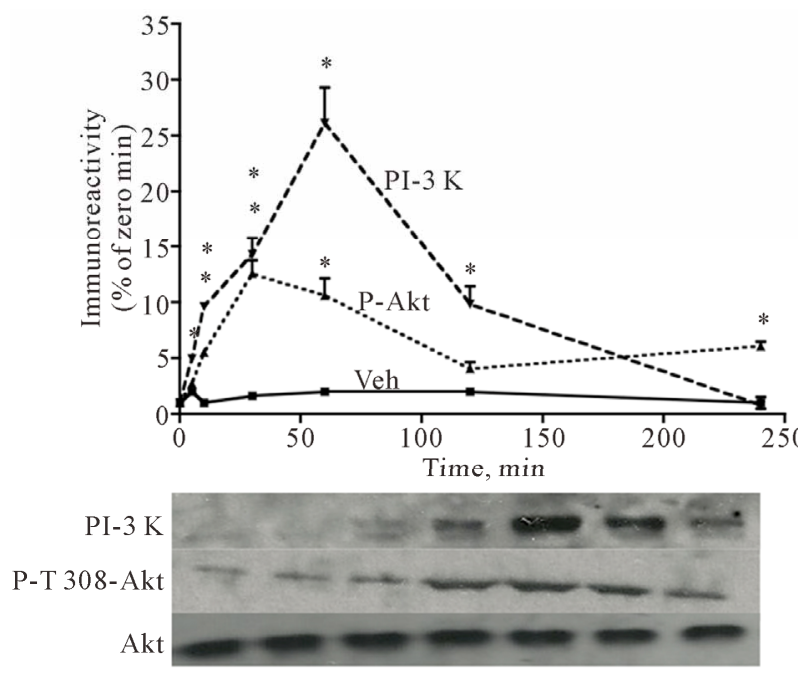

(a)
Phosphorylation of the transcription factor, CREB, exhibited no overall statistically significant difference among any of the time points with each other (Figure 3(b)).

Phosphorylation of neither MAPK 1 nor MAPK 2 was statistically significantly different over time (Figure 3(c)).

To verify that the signaling pathways evaluated were indeed affected by our treatments of one or both neurotransmitters, the appropriate pathway inhibitor was incubated with 5-HT +/- norepinephrine. LY 294002 or wortmannin inhibits the PI-3 K/Akt pathway, PD 98059 inhibits the MAPK pathway and K252a inhibits TrkB phosphorylation (Figure 3(d)).

\subsection{Kinetics of Activation of Intracellular Signaling in Hippocampal Cell Lysates in Response to both 5-HT and Norepinephrine}

Both the PI-3 K and MAPK pathways, as well as BDNF, TrkB and CREB, were differentially activated in response to application of 5-HT and norepinephrine over time (Figure 4). PI-3 K was significantly activated over time $\left[\mathrm{F}_{(6,14)}=257.18, p<0.000\right]$. PI-3 K expression was significantly higher at all time points than at zero $\min (p$ $<0.000)$; compared to that at zero min, PI-3 K expression was significantly higher at the 5- $(p=0.016), 60-(p=$ $0.006), 120-(p=0.003)$ and $240-(p<0.000)$ min time points. The 30-min time point expression of PI-3 K exhibited significantly less than that at any other time point, except for at zero- $(p<0.000)$ and at $240 \mathrm{~min}$, compared to which, it was statistically the same. Finally, at $60 \mathrm{~min}$,
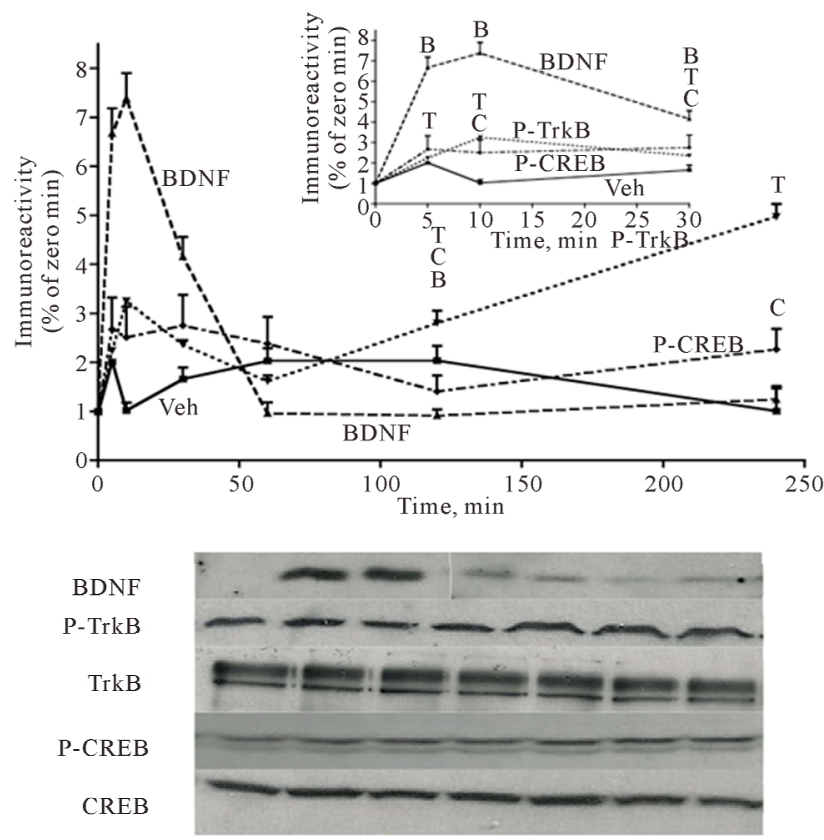

(b) 


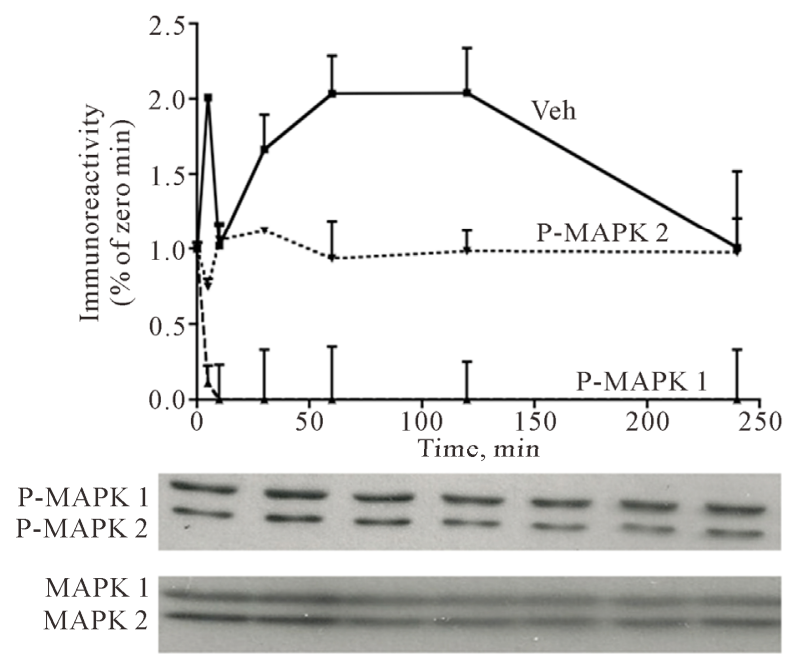

(c)
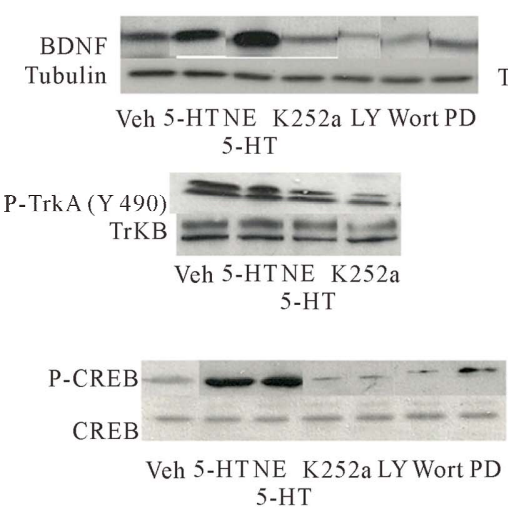

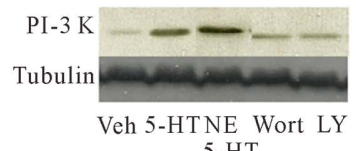

$5-\mathrm{HT}$

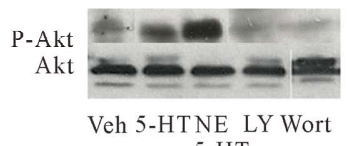
$5-\mathrm{HT}$

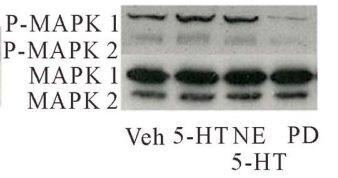

d)

Figure 3. Serotonin differentially affects various signaling intermediates and pathways. For ease of comparison, all 7 signaling molecules were divided into 3 graphs (a)-(c): (a) vehicle, PI-3 K and P-Akt, (b) vehicle, BDNF, P-TrkB and P-CREB, and (c) vehicle, P-MAPK 1 and P-MAPK 2. These 3 graphs are displayed to provide maximum clarity with respect to the ordinate and do not reflect the relative proportions of all 7 signaling molecules (P-Akt, PI-3 K, BDNF, P-TrkB, P-CREB, P-MAPK 1, P-MAK 2). Vehicle represents the mean of the responses of all 7 signaling molecules in response to application of vehicle, which serves as baseline; its small error bars reveal that there are no statistically significant differences among all 7 molecules and are displayed as such to help minimize complexity of the graphs. For such direct comparisons among all 7 molecules, a 2-way ANOVA was conducted (molecule $x$ time, see Methods). Levels of both P-MAPK 1 and P-MAPK 2 are significantly lower than levels of PI-3 K at $5(p=0.013-0.014), 10(p<0.000), 30(p=0.004-0.006), 60(p<0.000)$ and $120(p<$ 0.000) min; levels of both P-MAPK 1 and P-MAPK 2 are significantly higher than levels of P-Akt throughout to 240 min $(p<$ 0.000 - 0.043). Levels of P-CREB was significantly lower than those of PI-3 K at $5(p=0.001), 10(p=0.001), 30(p=0.008)$ and levels of P-Akt at $30 \mathrm{~min}(p=0.022), 60(p=0.001)$ and $120(p<0.000) \mathrm{min}$; levels of P-CREB were significantly higher than those of both P-MAPK 1 and P-MAPK 2 up to 60 min inclusive $(p=0.009-0.017)$. (a) Compared to baseline (vehicle), PI-3 K is significantly higher from $5-120 \mathrm{~min}(p<0.001)$. P-Akt is significantly higher than that of baseline from $10-240$ $\min (p<0.01)$; PI-3 K is significantly higher than P-Akt at $10(p=0.05), 60(p<0.000)$ and $120(p=0.001)$ min. *, statistically significantly different from zero time point at $p<0.05$; one column of two *, both PI-3 K and P-Akt are statistically significantly different from zero time point at $p<0.05$. (b) BDNF is significantly higher than that of baseline from $5-30$ min $(p<$ 0.05 , inset) and significantly lower than that of baseline vehicle at 60 and $120 \mathrm{~min}(p<0.05)$; P-TrkB is significantly higher than that of baseline vehicle at $10(p=0.043), 30(p=0.043), 120(p=0.045)$ and $240(p=0.012)$ min; P-CREB levels are significantly higher than that of baseline at only $30 \mathrm{~min}(p=0.026)$. BDNF is significantly higher than P-TrkB at $5(p=0.008), 10$ $(p=0.011)$ and $30(p=0.035) \mathrm{min}$; BDNF is significantly higher than P-CREB at $5(p=0.01)$ and $10(p=0.015)$ min; and $P$ TrkB is significantly higher than P-CREB at 120- $(p=0.016)$ and $240-(p=0.009)$ min. Inset: $B$, T and C refer to BDNF, PTrkB and P-CREB, respectively, and indicate that levels of this molecule are significantly different from that at zero min at $p$ 0.05. (c) Both P-MAPK1 and P-MAPK 2 levels are significantly lower than that of baseline from $30-120 \mathrm{~min}(p=0.004-$ 0.039). Levels of P-MAPK 1 and P-MAPK 2 did not significantly differ from each other throughout the entire duration. Data are the mean \pm SEM. (d) Representative immunoblots in the presence of the appropriate inhibitor for TrkB $(\mathrm{K252a}, 10 \mu \mathrm{M})$, PI-3 K/Akt pathway (LY, LY $294002(10 \mu M)$ or Wort, wortmannin $(20 \mu M))$ or MAPK (PD, PD $98059,50 \mu M)$ pathway. Each inhibitor was added at this final concentration $1 \mathrm{hr}$ prior to the application of neurotransmitter. Each experiment was conducted at least twice, with each sample measured in triplicate.

PI-3 K expression was significantly higher than at all other time points, except that at $10 \mathrm{~min}$ and $120 \mathrm{~min}$ (Figure 4(a)). Akt was significantly phosphorylated over time $\left[\mathrm{F}_{(6,14)}=72.07, p<0.000\right]$. Akt phosphorylation was significantly higher at all time points than at zero $\min (p<0.000)$; Akt phosphorylation was significantly higher at both the 5- and 10-min time points than at the 30 - and 240-min time points $(p<0.000)$. At $60 \mathrm{~min}$, Akt was phosphorylated significantly higher than at all other time points $(p<0.000-0.009)$, except those at 5 and 10 min. At $120 \mathrm{~min}$, phosphorylation of Akt was signifi- cantly higher than at 30 and $240 \min (p<0.000)$ statistically the same as that at $60 \min (p=0.009)$ (Figure 4(a)).

BDNF expression was significantly activated in response to application of 5-HT and norepinephrine over time $\left[\mathrm{F}_{(6,14)}=17.97, p<0.000\right]$, where the expression was significantly higher at $30(p<0.000), 120(p<0.000)$ and $240(p=0.001) \mathrm{min}$ and significantly lower at 60 $\min (p=0.000)$ than at zero min (Figure 4(b)). At both 5 and $10 \mathrm{~min}, \mathrm{BDNF}$ expression was significantly lower than that at $30(p=0.004,0.018$, respectively $), 120$ 

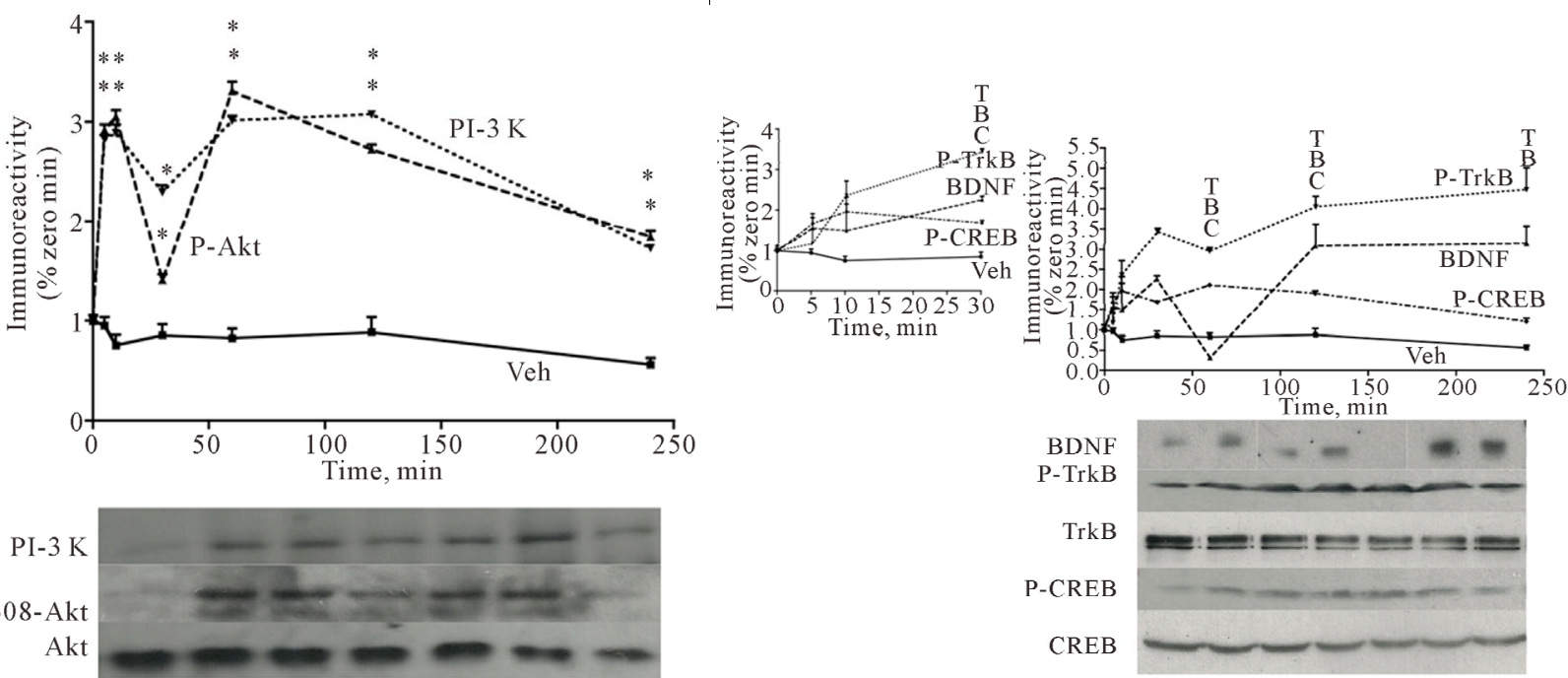

(a)

(b)
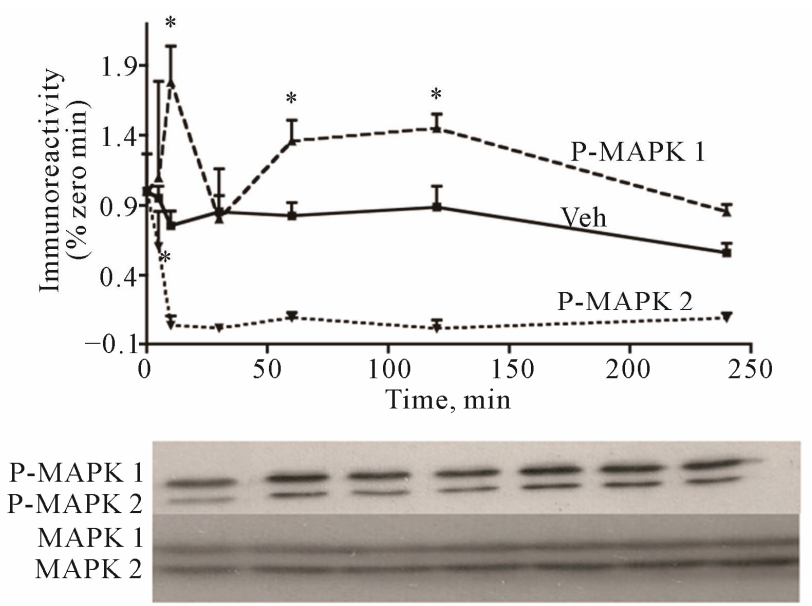

(c)

Figure 4. Serotonin and norepinephrine administered together differentially affect various signaling intermediates and pathways. For ease of comparison, all 7 signaling molecules were divided into 3 graphs (a)-(c): (a) vehicle, PI-3 K and P-Akt, (b) vehicle, BDNF, P-TrkB and P-CREB, and (c) vehicle, P-MAPK 1 and P-MAPK 2. These 3 graphs are displayed to provide maximum clarity with respect to the ordinate and do not reflect the relative proportions of all 7 signaling molecules $(\mathrm{P}-\mathrm{Akt}$, PI-3 K, BDNF, P-TrkB, P-CREB, P-MAPK1, P-MAPK 2). Vehicle represents the mean of the responses of all 7 signaling molecules in response to application of vehicle, which serves as baseline; its small error bars reveal that there are no statistically significant differences among all 7 molecules and are displayed as such to help minimize complexity of the graphs. For such direct comparisons among all 7 molecules, a 2-way ANOVA was conducted (molecule $x$ time, see Methods). Activity of both PI-3 K and P-Akt are significantly higher than that of both P-MAPK1 and P-MAPK $2(p=0.003-0.028)$, even at 30 min. P-Akt levels are significantly lower than that of BDNF $(p=0.022)$ and P-TrkB $(p<0.000)$, but is statistically the same as that as P-CREB at $30 \mathrm{~min}$; then, at $60 \mathrm{~min}$, P-Akt levels are significantly higher than that of BDNF $(p<0.000)$ and P-CREB $(p=$ 0.033), but not statistically different from that of P-TrkB; at 240 min, P-Akt is significantly lower than that of BDNF $(p=$ $0.012)$ and P-TrkB $(p=0.034)$, and significantly higher than that of P-CREB $(p=0.037)$. Finally, starting at 30 min, P-TrkB levels are significantly higher than that of both P-MAPK $1(p=0.004-0.039)$ and P-MAPK $2(p<0.000)$ throughout to 240 min; at $60 \mathrm{~min}$, BDNF levels are significantly lower than that of P-MAPK1 $(p=0.029)$, but significantly higher than that of PMAPK $2(p<0.000)$; but at 120 to 240 min, BDNF levels are significantly higher than that of both P-MAPK $1(p<0.001)$ and P-MAPK 2 ( $p<0.001)$. (a) Expression and activity levels of both P-Akt and PI-3 K, whose time courses parallel each other, are significantly higher than that of baseline (vehicle) from $5-240 \mathrm{~min}(p<0.001)$. *, statistically significantly different from zero time point at $\boldsymbol{p}<\mathbf{0 . 0 5}$; one column of two *, both PI-3 K and P-Akt are statistically significantly different from zero time point at $p<0.05$. (b) BDNF is significantly higher than that of baseline vehicle from $5-30 \mathrm{~min}(p<0.05$, inset) and statistically the same as that of baseline at $60 \mathrm{~min}$, but is significantly higher than that of baseline at $120(p=0.019)$ and $240(p=$ 0.024) min; P-TrkB is significantly higher than that of baseline throughout from $5-240 \mathrm{~min}(p=0.013-0.041)$; P-CREB is significantly higher than that of baseline throughout from $5-240 \mathrm{~min}(p=0.001-0.018)$. BDNF is significantly higher than 
that of P-CREB at $30(p=0.039), 120(p=0.041)$ and $240(p=0.02)$ min, but is significantly lower than that of the phosphorylated transcription factor at $60 \mathrm{~min}(p=0.013)$; BDNF is significantly lower than that of $P$-TrkB at $30(p=0.026), 60(p$ $=0.009), 120(p=0.045)$ and $240(p=0.038)$ min; P-TrkB levels are significantly higher than those of P-CREB starting at 30 $(p<0.001), 60(p=0.006), 120(p=0.005)$ and $240(p=0.002)$ min. Inset: B, T and C refer to BDNF, P-TrkB and P-CREB, respectively, and indicate that levels of this molecule are significantly different from that at zero min at $p<0.05$. (c) P-MAPK 1 levels are significantly higher than that of baseline at $10(p<0.001), 60(p=0.021), 120(p=0.031)$ and $240(p=0.048)$, but not at 5 nor $30 \mathrm{~min}$; P-MAPK 2 levels are significantly lower than that of baseline starting at 10 min throughout to 240 min $(p$ $<0.001$ ); likewise, P-MAPK1 levels are significantly higher than those of P-MAPK 2 starting at 10 min throughout to 240 min $(p<0.001)$. *, statistically significantly different from zero time point at $p<0.05$. Data are the mean \pm SEM. Each experiment was conducted at least twice, with each sample measured in triplicate.

$(p=0.000,0.000$, respectively $)$ and $240(p=0.013,0.05$, respectively). At $60 \mathrm{~min}$, BDNF expression was significantly lower than at all other time points $(p=0.000$ 0.004). At $240 \mathrm{~min}$, BDNF expression was significantly higher than that at zero and at $60 \mathrm{~min}(p=0.001)$ (Figure 4(b)).

Differences in phosphorylation of TrkB was significant over time $\left[\mathrm{F}_{(6,21)}=36.86, p<0.000\right]$ : all time points, except at $5 \mathrm{~min}$, saw a statistically significant increase in TrkB phosphorylation $(p<0.000)$ compared with that at zero min. However, starting at $10 \mathrm{~min}$, TrkB phosphorylation was not significantly different from all subsequent time points.

CREB phosphorylation was significantly different over time $\left[\mathrm{F}_{(6,14)}=30.59, p<0.000\right]$ in response to application of both 5 -HT and norepinephrine. Starting at zero min, all time points were significantly higher $(p<$ 0.000 ); at $5 \mathrm{~min}$, phosphorylation was significantly lower than at all other subsequent time points $(p<0.000$ $0.014)$, except at $30 \mathrm{~min}$; at $10 \mathrm{~min}$, phosphorylation was significantly higher $(p<0.000-0.014)$, except at 60 and $120 \mathrm{~min}$; at $30 \mathrm{~min}$, phosphorylation was significantly higher than that at zero and significantly lower than that at $10(p=0.008), 60(p<0.000)$ and $120(p=0.023) \mathrm{min}$ (Figure 4(b)).

Phosphorylation of MAPK 1 was significantly different over time $\left[\mathrm{F}_{(6,14)}=25.63, p<0.000\right]$ in response to application of both 5-HT and norepinephrine. Phosphorylation of MAPK 1 was significantly higher at the $10-(p=0.012), 60-(p=0.42)$ and $120-(p=0.039) \mathrm{min}$ time points than that at zero min (Figure 4(c)). Likewise, phosphorylation of MAPK 2 was significantly different over time in response to both neurotransmitters $\left[\mathrm{F}_{(6,14)}=\right.$ $6.22, p=0.0212]$. MAPK 2 phosphorylation was significantly higher at the $5-\min (p=0.039-0.009)$ than at all subsequent time points (Figure 4(c)).

\subsection{Kinetics of BDNF Concentrations in Lysates and Media in Response to Norepinephrine, Serotonin or both}

In response to application of norepinephrine, levels of BDNF between lysates and media were significantly changed over time $\left[\mathrm{F}_{(6,14)}=46.81, p<0.001\right]$ (Figure 5(a)). BDNF levels in lysates were significantly higher at
120 min than that $30(p=0.002)$ and $480(p=0.008) \mathrm{min}$ (Figure 5(a)). BDNF levels in media were significantly higher at $240 \mathrm{~min}(p<0.001)$ than at all other time points evaluated (Figure 5(a)).

In response to application of 5-HT, levels of BDNF between lysates and media were significantly changed over time $\left[\mathrm{F}_{(6,14)}=29.44, p<0.001\right]$ (Figure 5(b)) . BDNF levels in lysates were significantly higher at 10 $\min (p=0.007)$ than at all other time points. BDNF levels in media were significantly higher at $10(p<0.000)$ and $30(p<0.000)$ min than at all other time points evaluated (Figure 5(b)).

In response to application of both norepinephrine and 5-HT, levels of BDNF between lysates and media were significantly changed over time $\left[\mathrm{F}_{(6,14)}=18.76, p=\right.$ 0.002] (Figure 5(c)). BDNF levels in lysates were significantly higher at $10 \mathrm{~min}(p=0.004)$ and lower at 30 $\min (p=0.006)$ than at all other time points (Figure 5(c)). BDNF levels in media were significantly lower at $30 \min (p=0.039)$ than at all other time points evaluated (Figure 5(c)).

\section{Discussion}

We have previously shown that hippocampal neurons in culture respond to application of norepinephrine by increasing expression of BDNF, its receptor, TrkB, activation of the PI- $3 \mathrm{~K}$ and MAPK pathways, and the transcription factor, CREB [24]. In that study, we demonstrated that in a dose- and time-response experiment, $10^{-7}$ $\mathrm{M}$ norepinephrine for $2 \mathrm{hr}$ was maximally effective in eliciting BDNF expression; consistently, activation of TrkB was sustained for $4 \mathrm{hr}$ and that of PI-3 K and MAPK pathways was maintained out to $8 \mathrm{hr}[24,34]$. In the current study, we extend characterization of norepinephrine-induced expression of BDNF, not only within hippocampal neurons themselves (lysates via immunoblotting, [24]), but also, to the kinetics of BDNF release into the surrounding media (Figure 5(a)). We also characterized the kinetics of 5-HT-induced BDNF expression (Figure 5(b)) just as we did earlier with norepinephrine [24]. As expected, the time course expression of BDNF in neuronal lysates precedes that released into the media (Figures 5(a), (b)).

We show that although significant BDNF expression is 


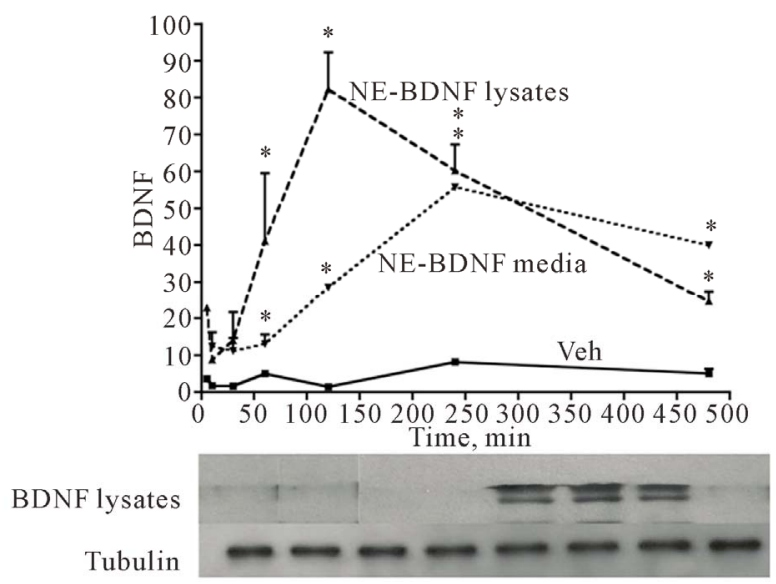

(a)

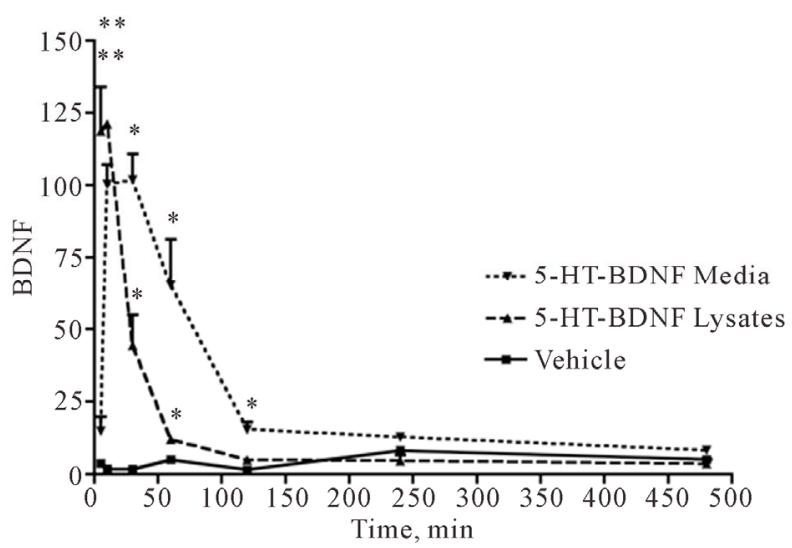

(b)

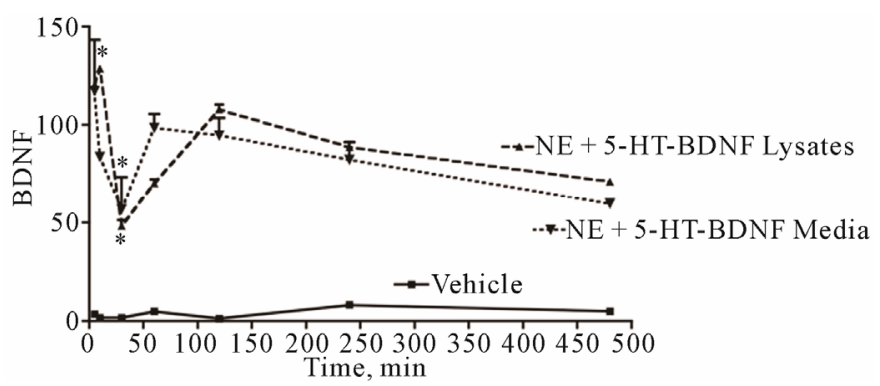

(c)

Figure 5. Norepinephrine and/or 5-HT increase BDNF in neuronal lysates, followed by an increase into the surrounding media. (a) Norepinephrine increased BDNF in both lysates $(p<0001)$ and media $(p<0.001)$ significantly higher than that in baseline vehicle controls at zero min. Lysate levels of BDNF are significantly higher than media levels at 120 min $(p<0.001)$ (b) Serotonin increased BDNF in both lysates $(p<0.001)$ and media $(p<0.001)$ significantly higher than that in baseline vehicle controls. Media levels of BDNF are significantly higher than lysate levels at $30(p=0.001), 60(p=0.034)$ and $120(p=$ 0.043) min. (c) Both norepinephrine and 5-HT increased BDNF in both lysates $(p=0.003)$ and media $(p=0.0025)$ significantly higher than that in baseline controls. Both neurotransmitters resulted in no significant differences in BDNF between lysates and media, except at $60 \mathrm{~min}(p=0.045)$. NE, norepinephrine; *, statistically significantly different from zero time point at $p<$ 0.05. Data are the mean \pm SEM. Each experiment was conducted at least twice, with each sample measured in triplicate.

indeed induced at the lowest concentration of 5-HT evaluated $\left(10^{-9} \mathrm{M}\right)$, 5-HT induces maximal BDNF expression on a much shorter time scale [32], but less potently $\left(10^{-5} \mathrm{M}\right.$, Figure 1) than that of norepinephrine [24]. Moreover, we show that norepinephrine-induced secretion of BDNF into the media manifests a delay of about $2 \mathrm{hr}$ (from 120 to $240 \mathrm{~min}$ ) from which expression of BDNF in neuronal lysates themselves was maximal at $120 \mathrm{~min}$; following another $2 \mathrm{hr}$, BDNF levels in the media were decreased to those comparable at $120 \mathrm{~min}$. A similar pattern of BDNF secretion into the media was observed with 5-HT; except that at 10 and $30 \mathrm{~min}, \mathrm{BDNF}$ was maximal and then decreased significantly $30 \mathrm{~min}$ later (at $60 \mathrm{~min}$ ), but was still higher than that at 5, 120, and $240 \mathrm{~min}$ (Figure 2). Again, this represents a delay between maximal BDNF expression observed in lysates at 1 and $10 \mathrm{~min}$, compared to that observed in the media at 10,30 and $60 \mathrm{~min}$ later.

Then, when norepinephrine and 5-HT are co-administered, BDNF secretion into the media is significantly higher throughout the duration of this time scale, out to $240 \mathrm{~min}$. Surprisingly, at both the beginning ( $5 \mathrm{~min}$ ) and at the end $(240 \mathrm{~min})$, BDNF secretion into the media indicates a synergistic effect between the 2 neurotransmiters as neither one alone elicited such a large response (Figure 2). Upon application of both neurotransmitters, the time course of BDNF in lysates and media roughly parallel each other (Figure 5(c)). Presented another way, when norepinephrine and 5-HT are co-administered, except for a momentary decline at $30 \mathrm{~min}$ in lysates, either neurotransmitter alone or both together result in increased BDNF expression (Figure 6(a)). This tendency is even more pronounced in the media in which striking synergistic effects are observed between norepinephrine and 5-HT (Figure 6(b)).

At first glance of the data, it would seem that when each neurotransmitter is added alone, 5-HT would increase BDNF early, whereas norepinephrine would increase it later. When co-administered, however, this tissue culture system does not respond so simplistically, 


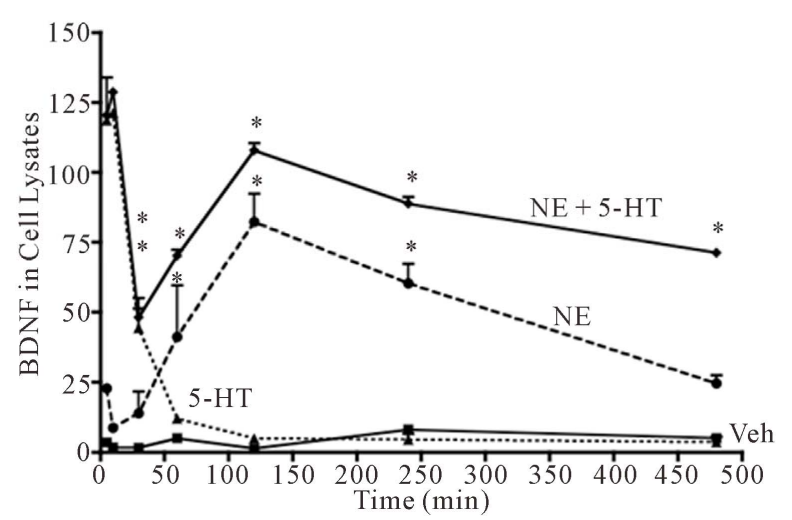

(a)

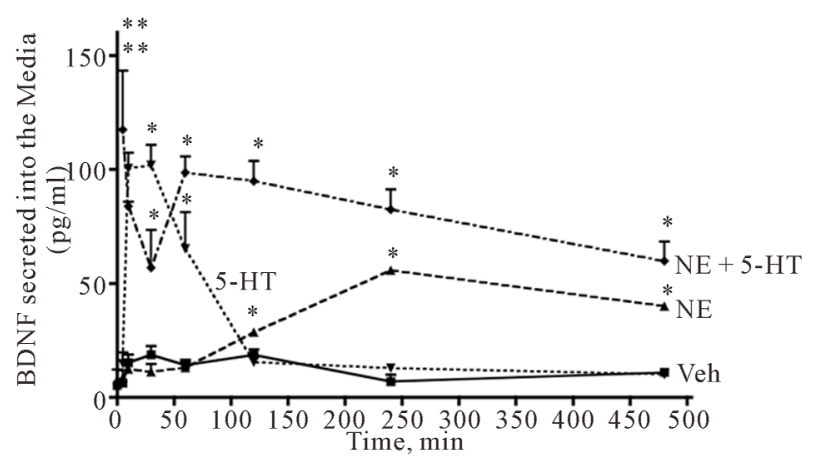

(b)

Figure 6. Norepinephrine and/or 5-HT increase BDNF in cell lysates and that secreted into the media over time. (a) BDNF in cell lysates is significantly higher as a result of norepinephrine or 5-HT administered separately or together, compared to that in baseline controls across all time points $(p=0.001-0.022)$; starting at $60 \mathrm{~min}$, BDNF levels are significantly higher as a result of both neurotransmitters than that as a result of only norepinephrine $(p=0.003$ $0.021)$ or only 5-HT $(p<0.001)$. Note the significant interaction with time, at $30(p=0.014)$ and $60(p=0.022) \mathrm{min}$, which shows BDNF levels increasing as a result of norepinephrine application and decreasing as a result of 5-HT application. one column of two *, both 5-HT and NE + 5-HT are statistically significantly different from zero time point at $p<0.05$. (b) BDNF in media is significantly higher as a result of 5-HT at $10(p<0.000), 30(p<0.000)$ and $60(p<$ $0.000)$ min than that at baseline; BDNF in media is significantly higher as a result of norephinephrine at 240 ( $p=$ $0.001)$ and $480(p=0.016)$ min than that at baseline; and BDNF in media is significantly higher as a result of both norepinephrine and 5-HT than that at baseline across all time points $(p<0.000)$. NE, norepinephrine; *, statistically significantly different from zero time point at $p<0.05$.

resulting in an overlap during which the entire time frame evaluated sees an increased expression of BDNF. Rather, such a momentary decline at 30 to 60 min belies a more complex mechanism, rather than simply the sum of the effects of two different neurotransmitters on BDNF expression and was so reliable, that the Westerns and ELISAs were each performed at least 4 - 5 times to confirm. This temporary decline is seen with BDNF in lysates (60 min, Figure 4(b); $30 \mathrm{~min}$, Figures 5(c), 6(a)), BDNF in media (30 min, Figures 2, 5(c), 6(b)), P-TrkB (60 min, Figure 4(b)), PI-3 K and P-Akt (30 min, Figure 4(a)), P-CREB (30 min, Figure 4(b)) and MAPK 1 (30 min, Figure 4(c)). It is conceivable that the co-presence of both neurotransmitters entails that each bind their respective GPCRs, resulting in production of cAMP and subsequent activation of PKA, which then phosphorylates CREB, which, then regulates the transcription of BDNF, which, in turn would be packaged into vesicles and be released extracellularly, where it would bind its TrkB receptors via an autocrine or paracrine diffusion [35], resulting in a positive feed-back loop [24]; all of this could occur within $5-10 \mathrm{~min}$ and certainly before 30 - $60 \mathrm{~min}$. Then, after 30 - $60 \mathrm{~min}$, this released BDNF can then modulate a rapidly recycling pool of vesicles storing norepinephrine and/or 5-HT by promoting the entry of $\mathrm{Ca}^{2+}$ and subsequent activation of the SNARE complex and consequent increased release of these neurotransmitters [36-38]. Such a positive feedback loop [24] is corroborated in Figure 3(d) in which PI-3 K/Akt and MAPK inhibitors result in decreased expression of BDNF and decreased phosphorylation of CREB.

In mixed hippocampal cultures (neurons and astrocytes), once they have begun establishing some rudimentary connections, the application of neurotransmitter may be considered as roughly equivalent to the application of neurotransmitter-specific antidepressant, such as reboxtine (norepinephrine-specific reuptake inhibitor) or fluoxetine (serotonin-specific reuptake inhibitor). Indeed, treating cultured astrocytes with fluoxetine [39], cultured cortical neurons with imipramine or fluvoxamine [5], or mixed cultured hippocampal neurons/astrocytes with reboxetine or tranylcypromine [24] have all led to increased expression of BDNF. Antidepressants that work through both neurotransmitters, therefore, would not only release BDNF faster, but for longer periods of time. To resolve the apparent discrepancy between in vivo efficacy and in vitro potency, Tsuruda et al. [40] characterized the in vitro binding kinetics of several antidepressants in HEK 293 cells and found a wide range of kinetics binding affinities among many of the currently used antidepressants, thereby underscoring the need for standard assays and cell lines to screen and consistently characterize antidepressants in terms of their ability to exert long-term cellular and genetic effects. Even in our lowdensity culture of $50 \mathrm{~K} / \mathrm{cm}^{2}$, which is considerably lower than those screened by others [35] at $80 \mathrm{~K} / \mathrm{cm}^{2}$, BDNF exhibits both autocrine and paracrine diffusive radii [35], as evidenced by the activation of TrkB, PI-3 K and MAPK pathways and CREB. Indeed, we have found that the PI-3 K/Akt pathway can be activated as early as 10 min following application of 5-HT (Figure 3(a)). This is 
consistent with earlier findings of $10^{-8} \mathrm{M} 5$-HT within as little as 2 to $5 \min$ [32] and with what others found, albeit in cerebellar granule neurons, that direct application of BDNF itself resulted in phosphorylation of Akt in as little as $5 \mathrm{~min}$ and was sustained for $6 \mathrm{~h} \mathrm{[41].} \mathrm{To} \mathrm{achieve}$ rapid and sustained activation of these survival signaling pathways, therefore, if not applying BDNF directly $[32,41]$, but rather, using antidepressants or the neurotransmitters themselves, at least both the noradrenergic and serotonergic systems must be targeted. This latter indirect application of BDNF provides further evidence for receptor/pathway crosstalk between GPCRs and Trk receptors ([24], and references cited therein; [42,43]).

The time course for increased BDNF expression and release into the media is consistent with earlier studies that found increased BDNF kinetics on the order of several minutes [44]. High $\mathrm{K}^{+}$concentration has been shown in cultured hippocampal neurons to secrete both pro- and mature BDNF for $90 \mathrm{~min}$ following $\mathrm{K}^{+}$-induced depolarization [17], which is comparable to our findings in response to norepinephrine-induced BDNF secretion starting at $120 \mathrm{~min}$. This suggests that depolarizationdriven BDNF release, when it occurs slowly (longer time scale) may be mediated by noradrenergic receptor binding. Faster BDNF release (or shorter time scale) may be mediated serotonergically as the current study shows. Shen et al. [45] have also shown in vivo, that in as little as 15 min of swim stress, hippocampal P-MEK is dramatically increased. Chen and Russo-Neustadt [8] have shown that a single overnight bout of running exercise significantly increased hippocampal BDNF and P-CREB. Thus, although Aicardi et al. [46] found that in peripheral cortical slices, theta burst stimulation at a frequency known to induce LTP, the subsequent secretion of BDNF was sustained $5-12$ min beyond the stimulation period. High $\mathrm{K}^{+}$has also been shown to result in rapid BDNF release (400 sec, [22]). It is possible that these few minutes beyond the stimulation interval are serotonergically mediated.

Still, it is not clear why this discrepancy in kinetics of release may exist between noradrenergically and serotonergically mediated release of BDNF. Others have suggested that the speed of BDNF release/secretion is limited by the slow dissolution of the secretory granule peptide core following opening of the fusion pore connecting the membranes of the granule with that of the cell [47]. It is also possible that co-localization of BDNF with other small peptides in the secretory granule in both pre- and post-synaptic terminals [48], their respective concentrations and molecular weights, their speed of binding and unbinding from the core and their speed of diffusion through the fusion pore all determine the rate of secretion into the media [47]. Moreover, secretion of BDNF is relegated to the regulated pathway of secretion and co-localization with the PSD 95 in glutamatergic synapses [49]. Those investigators found that depolarization-induced release of BDNF from secretory granules typically takes the longest of all neurotrophins, on the order of about $300 \mathrm{sec}[13,49]$, following a 30 -sec delay onset and a half-life decay of about $230 \mathrm{sec}$ [49]. Finally, there is ample evidence that because BDNF is actually secreted in its precursor form (pro-BDNF), which is then cleared from the extracellular space via rapid uptake by nearby astrocytes and neurons, which internalize proBDNF via formation with a complex with the $\mathrm{p} 75$ receptor and subsequent endocytosis. This endocytosed pro-BDNF is then routed to a fast recycling pathway for subsequent soluble NSF attachment protein receptordependent secretion occurring on a $5-\mathrm{min}[50]$ to $10-\mathrm{min}$ [16] time scale.

\section{Conclusion}

In conclusion, our hippocampal culture study shows that norepinephrine and 5-HT differentially induce the expression and release of BDNF. Serotonin elicits an earlier, but less sustained expression and release of BDNF, whereas norepinephrine elicits more delayed and more sustained release of the neurotrophin. Together, both neurotransmitters cover the entire time scale evaluated. In addition, for each neurotransmitter, the amount of BDNF in cell lysates shortly preceded those in the media. Consistent with their induced expression of BDNF, activation of TrkB, CREB and the PI-3 K/Akt, but not the MAPK pathway was sustained throughout the time course evaluated. These results, taken together along with those of our earlier study [24], provide a further insight into the possible mechanisms of neurotransmitter action such as that which occurs with physical exercise and/or antidepressant therapy.

\section{REFERENCES}

[1] A. Gärtner and V. Staiger, "Neurotrophin Secretion from Hippocampal NeuronsEvoked by Long-Term-Potentiation-Inducing Electrical Stimulation Patterns," Proceedings of the National Academy of Sciences USA, Vol. 99, No. 9, 2002, pp. 6386-6391. http://dx.doi.org/10.1073/pnas.092129699

[2] M. E. Hopkins, R. Nitecki and D. J. Bucci, "Physical Exercise during Adolescence versus Adulthood: Differential Effects on Object Recognition Memory and BDNF Levels," Neuroscience, Vol. 194, 2011, pp. 84-94. http://dx.doi.org/10.1016/j.neuroscience.2011.07.071

[3] T. Kobilo, Q-R. Liu, K. Gandhi, M. Mughal, Y. Shaham and H. van Praag, "Running Is the Neurogenic and Neurotrophic Stimulus in Environmental Enrichment," Learning \& Memory, Vol. 18, No. 9, 2011, pp. 605-609. http://dx.doi.org/10.1101/lm.2283011

[4] G. Baj, V. D’Alessandro, L. Musazzi, A. Mallei, C. R. 
Sartori, M. Sciancalepore, D. Tardito, F. Langone, M. Popoli and E. Tongiorgi, "Physical Exercise and Antidepressants Enhance BDNF Targeting in hippocampal CA3 Dendrites: Further Evidence of a Spatial Code for BDNF Splice Variants," Neuropsychopharmacology, Vol. 37, No. 7, 2012, pp. 1600-1611. http://dx.doi.org/10.1038/npp.2012.5

[5] Y. Yagasaki, T. Numakawa, E. Kumamaru, T. Hayashi, T.-P. Su and H. Kunugi, "Chronic Antidepressants Potentiate via Sigma-1 Receptors the Brain-Derived Neurotrophic Factor-Induced Signaling for Glutamate Release," Journal of Biological Chemistry, Vol. 281, No. 18, 2006, pp. 12941-12949.

http://dx.doi.org/10.1074/jbc.M508157200

[6] P. Rasmussen, P. Brassard, H. Adser, M. V. Pedersen, L. Leick, E. Hart, N. H. Secher, B. K. Pedersen and H. Pilegaard, "Evidence for a Release of Brain-Derived Neurotrophic Factor from the Brain During Exercise," Experimental Physiology, Vol. 94, No. 10, 2009, pp. 1062-1069. http://dx.doi.org/10.1113/expphysiol.2009.048512

[7] A. A. Russo-Neustadt and M. J. Chen, "Brain-Derived Neurotrophic Factor and Antidepressant Activity," Current Pharmaceutical Design, Vol. 11, No. 12, 2005, pp. 1495-1510. http://dx.doi.org/10.2174/1381612053764788

[8] M. J. Chen, A. A. Russo-Neustadt, "Running Exercise-Induced Up-Regulation of Hippocampal Brain-Derived Neurotrophic Factor is CREB-Dependent," Hippocampus, Vol. 19, No. 10, 2009, pp. 962-972. http://dx.doi.org/10.1002/hipo.20579

[9] M. C. Lee, M. Okamoto, Y. F. Liu, K. Inoue, T. Matsui, H. Nogami and H. Soya, "Voluntary Resistance Running with Short Distance Enhances Spatial Memory Related to Hippocampal BDNF Signaling," Journal of Applied Physiology, Vol. 113, No. 8, 2012, pp. 1260-1266. http://dx.doi.org/10.1152/japplphysiol.00869.2012

[10] A. Balkowiec and D. M. Katz, "Cellular Mechanisms Regulating Activity-Dependent Release of Native BrainDerived Neurotrophic Factor from Hippocampal Neurons," Journal of Neuroscience, Vol. 22, No. 23, 2002, pp. 10399-10407.

[11] F. Zafra, D. Lindholm, E. Castrén, J. Hartikka, and H. Thoenen, "Regulation of Brain-Derived Neurotrophic Factor and Nerve Growth Factor mRNA in Primary Cultures of Hippocampal Neurons and Astrocytes," Journal of Neuroscience, Vol. 12, No. 12, 1992, pp. 4793-4799.

[12] A. Androutsellis-Theotokis, W. J. McCormack, H. F. Bradford, G. M. Stern and F. B. Pliego-Rivero, "The Depolarisation-Induced Release of $\left[{ }^{125} \mathrm{I}\right] \mathrm{BDNF}$ from Brain Tissue," Brain Research, Vol. 743, No. 1-2, 1996, pp. 4048. http://dx.doi.org/10.1016/S0006-8993(96)00981-X

[13] M. Hartmann, R. Heumann and V. Lessmann, "Synaptic Secretion of BDNF after High-Frequency Stimulation of Glutamatergic Synapses," EMBO Journal, Vol. 20, No. 21, 2001, pp. 5887-5897. http://dx.doi.org/10.1093/emboj/20.21.5887

[14] M. Kojima, N. Takei, T. Numakawa, Y. Ishikawa, S. Suzuki, T. Matsumoto, R. Katoh-Semba, H. Nawa and H. Hatanaka, "Biological Characterization and Optical Imaging of Brain-Derived Neurotrophic Factor-Green Fluo- rescent Protein Suggest an Activity-Dependent Local Release of Brain-Derived Neurotrophic Factor in Neurites of Cultured Hippocampal Neurons," Journal of Neuroscience Research, Vol. 64, No. 1, 2001, pp. 1-10. http://dx.doi.org/10.1002/jnr.1080

[15] J. Goggi, I. A. Pullar, S. L. Carney and H. F. Bradford, "The Control of $\left[{ }^{125} \mathrm{I}\right] \mathrm{BDNF}$ Release from Striatal Rat Brain Slices," Brain Research, Vol. 967, No. 1-2, 2003, pp. 201-209.

http://dx.doi.org/10.1016/S0006-8993(03)02225-X

[16] S. Santi, S. Cappello, M. Riccio, M. Bergami, G. Aicardi, U. Schenk, M. Matteoli and M. Canossa, "Hippocampal Neurons Recycle BDNF for Activity-Dependent Secretion and LTP Maintenance," The EMBO Journal, Vol. 25, No. 18, 2006, pp. 4372-4380. http://dx.doi.org/10.1038/sj.emboj.7601303

[17] J. Yang, C.-J. Siao, G. Nagappan, T. Marinic, D. Jing, K. McGrath, Z.-Y. Chen, W. Mark, L. Tessarollo, F. S. Lee, B. Lu and B. L. Hempstead, "Neuronal Release of ProBDNF," Nature Neuroscience, Vol. 12, No. 2, 2009, pp. 113-115. http://dx.doi.org/10.1038/nn.2244

[18] A. M. Marini, S. J. Rabin, R. H. Lipsky and I. Mocchetti, (1998) “Activity-Dependent Release of Brain-Derived Neurotrophic Factor Underlies the Neuroprotective Effect of N-Methyl-D-Aspartate," Journal of Biological Chemistry, Vol. 273, No. 45, 1998, pp. 29394-29399. http://dx.doi.org/10.1074/jbc.273.45.29394

[19] R. H. Lipsky, K. Xu, D. Zhu, C. Kelly, A. Terhakopian, A. Novelli and A. M. Marini, "Nuclear Factor Kappa B is a Critical Determinant in $N$-Methyl-D-Aspartate Receptor-Mediated Neuroprotection," Journal of Neurochemistry, Vol. 78, No. 2, 2001, pp. 254-264. http://dx.doi.org/10.1046/j.1471-4159.2001.00386.x

[20] X. Wu, D. Zhu, X. Jiang, P. Okagaki, K. Mearow, G. Zhu, S. McCall, K. Banaudha, R. H. Lipsky and A. M. Marini, "AMPA Protects Cultured Neurons against Glutamate Excitotoxicity through a Phosphatidylinositol 3-KinaseDependent Activation in Extracellular Signal-Regulated Kinase to Upregulate BDNF Gene Expression," Journal of Neurochemistry, Vol. 90, No. 4, 2004, pp. 807-818. http://dx.doi.org/10.1111/j.1471-4159.2004.02526.x

[21] X. Jiang, F. Tian, K. Mearow, P. Okagaki, R. H. Lipsky and A. M. Marini, "The Excitoprotective Effect of $N$-Methyl-D-Aspartate Receptors is Mediated by a BrainDerived Neurotrophic Factor Autocrine Loop in Cultured Hippocampal Neurons," Journal of Neurochemistry, Vol. 94, No. 3, 2005, pp. 713-722. http://dx.doi.org/10.1111/j.1471-4159.2005.03200.x

[22] R. Kolarow, T. Brigadski and V. Lessmann, "Postsynaptic Secretion of BDNF and NT-3 from Hippocampal Neurons Depends on Calcium-Calmodulin Kinase II Signaling and Proceeds via Delayed Fusion Pore Opening," Journal of Neuroscience, Vol. 27, No. 39, 2007, pp. 10350-10364. http://dx.doi.org/10.1523/JNEUROSCI.0692-07.2007

[23] E. C. Gunther, C. S. Von Bartheld, L. J. Goodman, J. E. Johnson and M. Bothwell, "The G-Protein Inhibitor, Pertussis Toxin, Inhibits the Secretion of Brain-Derived Neurotrophic Factor," Neuroscience, Vol. 100, No. 3, 2000, pp. 569-579. 
http://dx.doi.org/10.1016/S0306-4522(00)00309-2

[24] M. J. Chen, T. V. Nguyen, C. J. Pike and A. A. RussoNeustadt, "Norepinephrine Induces BDNF and Activates the PI-3K and MAPK Cascades in Embryonic Hippocampal Neurons," Cellular Signaling, Vol. 19, No. 1, 2007, pp. 114-128.

http://dx.doi.org/10.1016/j.cellsig.2006.05.028

[25] N. J. Patel, M. J. Chen and A. A. Russo-Neustadt, "Norpinephrine and Nitric Oxide Promote Cell Survival Signaling in Hippocampal Neurons," European Journal of Pharmacology, Vol. 633, No. 1-3, 2010, pp. 1-9. http://dx.doi.org/10.1016/j.ejphar.2010.01.012

[26] D. Yang, M. Chen and A. Russo-Neustadt, "Antidepressants are Neuroprotective Against Nutrient Deprivation Stress in Hippocampal Neurons," European Journal of Neuroscience, Vol. 36, No. 5, 2012, pp. 2573-2587. http://dx.doi.org/10.1111/j.1460-9568.2012.08187.x

[27] R. K. Dishman, K. J. Renner, J. E. Whitte-Welkley, K. A. Burke, B. N. Bunnell, "Treadmill Exercise Training Augments Brain Norepinephrine Response to Familiar and Novel Stress," Brain Research Bulletin, Vol. 52, No. 5, 2000, pp. 337-342. http://dx.doi.org/10.1016/S0361-9230(00)00271-9

[28] J. F. Cryan, O. F. O’Leary, S. H. Jin, J. C. Friedland, M. Ouyang, B. R. Hirsch, M. E. Page, A. Dalvi,S. A. Thomas and I. Lucki, "Norepinephrine-Deficient Mice Lack Responses to Antidepressant Drugs, Including Selective Serotonin Reuptake Inhibitors," Proceedings of the National Academy of Sciences USA, Vol. 101, No. 21, 2004, pp. 8186-8191. http://dx.doi.org/10.1073/pnas.0401080101

[29] D. J. Nutt, "The Neuropharmacology of Serotonin and Norepinephrine in Depression," International Clinical Psychopharmacology, Vol. 17, Suppl. 1, 2002, pp. S1S12. http://dx.doi.org/10.1097/00004850-200206001-00002

[30] J. E. Malberg and J. A. Blendy, "Antidepressant Action: To the Nucleus and Beyond," Trends in Pharmacological Sciences, Vol. 26, No. 12, 2005, pp. 631-638. http://dx.doi.org/10.1016/j.tips.2005.10.005

[31] G. A. Banker and W. M. Cowan, "Rat Hippocampal Neurons in Dispersed Cell Culture," Brain Research, Vol. 126, No. 3, 1977, pp. 397-442. http://dx.doi.org/10.1016/0006-8993(77)90594-7

[32] D. S. Cowan, N. N. Farley-Johnson and T. Travkina, "5-HT $1 \mathrm{~A}$ Receptors Couple to Activation of Akt, but not Extracellular-Regulated Kinase (ERK) in Cultured Hippocampal Neurons," Journal of Neurochemistry, Vol. 93, No. 4, 2005, pp. 910-917. http://dx.doi.org/10.1111/j.1471-4159.2005.03107.x

[33] U. K. Laemmli, "Cleavage of Structural Proteins During the Assembly of the Head of Bacteriophage T4," Nature, Vol. 227, No. 5259, 1970, pp. 680-685. http://dx.doi.org/10.1038/227680a0

[34] A. Barnea, J. Roberts and S. D. Croll, "Continuous Exposure to Brain-Derived Neurotrophic Factor is Required for Persistent Activation of TrkB Receptor, the ERK Signaling Pathway, and the Induction of Neuropeptide Y Production in Cortical Cultures," Brain Research, Vol. 1020, Vol. 1-2, 2004, pp. 106-117.
[35] D. Lindholm, P. Carroll, G. Tzimagiorgis and H. Thoenen, "Autocrine-Paracrine Regulation of Hippocampal Neuron Survival by IGF-1 and the Neurotrophins BDNF, NT-3 and NT-4," European Journal of Neuroscience, Vol. 8, No. 7, 1996, pp. 1452-1460. http://dx.doi.org/10.1111/j.1460-9568.1996.tb01607.x

[36] W. J. Tyler and L. D. Pozzo-Miller, "BDNF Enhances Quantal Neurotransmitter Release and Increases the Number of Docked Vesicles at the Active Zones of Hippocampal Excitatory Synapses," Journal of Neuroscience, Vol. 21, No. 12, 2001, pp. 4249-4258.

[37] W. J. Tyler, S. P. Perrett and L. D. Pozzo-Miller, "The Role of Neurotrophins in Neurotransmitter Release," The Neuroscientist, Vol. 8, No. 6, 2002, pp. 524-531. http://dx.doi.org/10.1177/1073858402238511

[38] W. J. Tyler, X.-L. Zhang, K. Hartman, J. Winterer, W. Muller, P. K. Stanton and L. Pozzo-Miller, "BDNF Increases Release Probability and the Size of a Rapidly Recycling Vesicle Pool within Rat Hippocampal Excitatory Synapses," Journal of Physiology, Vol. 574, No. 3, 2006, pp. 787-803.

http://dx.doi.org/10.1113/jphysiol.2006.111310

[39] I. Alleman, H. Fiumelli, P. J. Magistretti and J.-L. Martin, "Fluoxetine Regulates the Expression of Neurotrophic/Growth Factors and Glucose Metabolism in Astrocytes," Psychopharmacology, Vol. 216, No. 1, 2011, pp. 75-84. http://dx.doi.org/10.1007/s00213-011-2190-y

[40] P. R. Tsuruda, J. Yung, W. J. Martin, R. Chang, N. Mai and J. A. M. Smith, "Influence of Ligand Binding Kinetics on Functional Inhibition of Human Recombinant Serotonin and Norepinephrine Transporters," Journal of Pharmacological and Toxicological Methods, Vol. 61, No. 2, 2010, pp. 192-204. http://dx.doi.org/10.1016/j.vascn.2009.12.003

[41] D. Zhu, R. H. Lipsky and A. M. Marini, "Co-Activation of the Phosphatidylinositol-3-Kinase/Akt Signaling Pathway by N-Methyl-D-Aspartate and TrkB Receptors in Cerebellar Granule Cell Neurons," Amino Acids, Vol. 23, No. 1-3, 2002, pp. 11-17.

http://dx.doi.org/10.1007/s00726-001-0103-9

[42] H. Yano and M. V. Chao, "Neurotrophin Receptor Structure and Interactions," Pharmaceutica Acta Helvetiae, Vol. 74, No. 2-3, 2000, pp. 253-260.

http://dx.doi.org/10.1016/S0031-6865(99)00036-9

[43] Q. Ma, "Beneficial Effects of Moderate Voluntary Physical Exercise and Its Biological Mechanisms on Brain Health," Neuroscience Bulletin, Vol. 24, No. 4, 2008, pp. 265-270. http://dx.doi.org/10.1007/s12264-008-0402-1

[44] V. Lessmann, K. Gottmann and M. Malcangio, "Neurotrophin Secretion: Current Facts and Future Prospects," Progress in Neurobiology, Vol. 69, No. 5, 2003, pp. 341374. http://dx.doi.org/10.1016/S0301-0082(03)00019-4

[45] C.-P. Shen, Y. Tsimberg, C. Salvadore and E. Meller, "Activation of Erk and JNK MAPK Pathways by Acute Swim Stress in Rat Brain Regions," BMC Neuroscience, Vol. 5, 2004, pp. 36.

http://dx.doi.org/10.1186/1471-2202-5-36

[46] G. Aicardi, E. Argilli, S. Cappello, S. Santi, M. Riccio, H. Thoenen and M. Canossa, "Induction of Long-Term Po- 
tentiation and Depression is Reflected by Corresponding Changes in Secretion of Endogenous Brain-Derived Neurotrophic Factor," Proceedings of the National Academy of Sciences of the United States of America, Vol. 101, No. 44, 2004, pp. 15788-15792. http://dx.doi.org/10.1073/pnas.0406960101

[47] V. Lessmann and T. Brigadski, "Mechanisms, Locations, and Kinetics of Synaptic BDNF Secretion: An Update," Neuroscience Research, Vol. 65, No. 1, 2009, pp. 11-22. http://dx.doi.org/10.1016/j.neures.2009.06.004

[48] I. Buldyrev, N. M. Tanner, H.-Y. Hsieh, E. G. Dodd, L. T. Nguyen and A. Balkowiec, "Calcitonin Gene-Related Peptide Enhances Release of Native Brain-Derived Neurotrophic Factor from Trigeminal Ganglion Neurons," Journal of Neurochemistry, Vol. 99, No. 5, 2006, pp.
1338-1350.

http://dx.doi.org/10.1111/j.1471-4159.2006.04161.x

[49] T. Brigadski, M. Hartmann and V. Lessmann, "Differential Vesicular Targeting and Time Course of Synaptic Secretion of the Mammalian Neurotrophins," Journal of Neuroscience, Vol. 25, No. 33, 2005, pp. 7601-7614. http://dx.doi.org/10.1523/JNEUROSCI.1776-05.2005

[50] M. Bergami, S. Santi, E. Formaggio, C. Cagnoli, C. Verderio, R. Blum, B. Berninger, M. Matteoli and M. Canossa, "Uptake and Recycling of Pro-BDNF for Transmitter-Induced Secretion by Cortical Astrocytes," Journal of Cell Biology, Vol. 183, No. 2, 2008, pp. 213-221. http://dx.doi.org/10.1083/jcb.200806137 